\title{
Atuação do enfermeiro no preparo para o parto normal e nascimento no contexto da atenção básica: revisão integrativa
}

Nurses' role in preparing for childbirth and birth in the context of primary care: an integrative review

\author{
El papel de la enfermera en la preparación para el parto y el parto normales en el contexto de la \\ atención primaria: una revisión integradora
}

Recebido: 24/07/2021 | Revisado: 01/08/2021 | Aceito: 04/08/2021 | Publicado: 09/08/2021

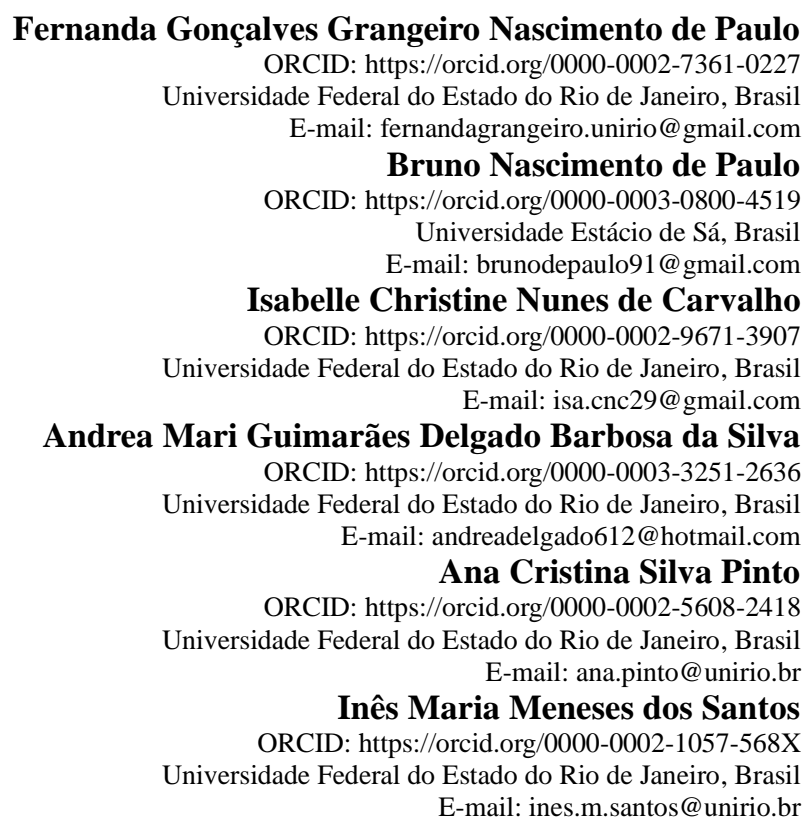

\begin{abstract}
Resumo
Objetivo: Analisar, em produção científica, ações de enfermagem que contribuem para a preparação da mulher gestante ao parto e nascimento. Método: Revisão integrativa, como questão: como a literatura aborda as ações do enfermeiro para a preparação da mulher gestante ao parto e nascimento? O levantamento ocorreu nas bases LILACS, BDENF e MEDLINE. Critérios de inclusão: pesquisa científica; idiomas português, inglês e espanhol; disponível na íntegra e online; e, publicados entre 2008 a 2018. A organização das informações permitiu a síntese, categorização e interpretação dos dados. Do cruzamento dos descritores totalizou 339 artigos; 49 foram excluídas por duplicação. Ao realizar a leitura dos 290 restantes, foram identificados 7 artigos para apreciação na íntegra. Após realizar a leitura e análise quanto à temática proposta, selecionaram-se três artigos. Como estratégia de sistematização, estruturou-se o quadro: caracterização das produções, categorização do estudo e ações do enfermeiro. Resultados: Foram publicados três artigos em 2013, 2017 e 2018 por profissionais apresentando vínculo com instituição de pesquisa. Foi construída uma categoria analítica: atividades educativas em saúde para o preparo do parto. Destacam-se como ações do enfermeiro: orientações quanto sinais de trabalho de parto, tecnologia do cuidado e da escolha do parto pela gestante e oficinas educativas com dramatizações relacionadas à gestação e ao trabalho de parto. Conclusão: As produções científicas estão centradas nas ações no que tange a fisiologia do parto. Nos artigos pesquisados não houve destaque para aspectos emocionais e de mudanças corporais do período gestatório até ao processo de parturição e a participação do acompanhante.
\end{abstract}

Palavras-chave: Cuidado pré-natal; Atenção primária à saúde; Enfermagem; Parto; Educação em saúde.

\footnotetext{
Abstract

Objective: To analyze, in scientific production, nursing actions that contribute to the preparation of pregnant women for childbirth and birth. Method: Integrative review, as a question: how does the literature address the actions of nurses to prepare pregnant women for childbirth and birth? The survey took place in the LILACS, BDENF and
} 
MEDLINE databases. Inclusion criteria: scientific research; Portuguese, English and Spanish languages; available in full and online; and, published between 2008 to 2018. The organization of information allowed the synthesis, categorization and interpretation of data. The crossing of descriptors totaled 339 articles; 49 were excluded by duplication. When reading the remaining 290, 7 articles were identified for full consideration. After reading and analyzing the proposed theme, three articles were selected. As a systematization strategy, the framework was structured: characterization of productions, categorization of the study and actions of the nurse. Results: Three articles were published in 2013, 2017 and 2018 by professionals with a relationship with a research institution. An analytical category was constructed: educational activities in health for childbirth preparation. The actions of the nurse stand out: guidelines regarding signs of labor, technology of care and the choice of childbirth by the pregnant woman and educational workshops with dramatizations related to pregnancy and labor. Conclusion: The scientific productions are centered on actions regarding the physiology of childbirth. In the researched articles, there was no emphasis on emotional aspects and bodily changes from the gestation period to the parturition process and the participation of the companion.

Keywords: Prenatal care; Primary health care; Nursing; Childbirth; Health education.

\section{Resumen}

Objetivo: Analizar, en la producción científica, las acciones de enfermería que contribuyen a la preparación de la gestante para el parto y el parto. Método: Revisión integradora, como pregunta: ¿cómo aborda la literatura las acciones de las enfermeras para preparar a las gestantes para el parto y el parto? La encuesta se realizó en las bases de datos LILACS, BDENF y MEDLINE. Criterios de inclusión: investigación científica; Idiomas portugués, inglés y español; disponible en su totalidad y en línea; y, publicado entre 2008 y 2018. La organización de la información permitió la síntesis, categorización e interpretación de datos. El cruce de descriptores totalizó 339 artículos; 49 fueron excluidos por duplicación. Al leer los 290 restantes, se identificaron 7 artículos para su consideración completa. Luego de leer y analizar el tema propuesto, se seleccionaron tres artículos. Como estrategia de sistematización, se estructuró el marco: caracterización de producciones, categorización del estudio y acciones del enfermero. Resultados: Se publicaron tres artículos en 2013, 2017 y 2018 por profesionales vinculados a una institución de investigación. Se construyó una categoría analítica: actividades educativas en salud para la preparación del parto. Se destacan las acciones de la enfermera: pautas en cuanto a signos de parto, tecnología de atención y elección del parto por parte de la gestante y talleres educativos con dramatizaciones relacionadas con el embarazo y el parto. Conclusión: Las producciones científicas se centran en acciones relacionadas con la fisiología del parto. En los artículos investigados, no hubo énfasis en los aspectos emocionales y cambios corporales desde el período gestacional hasta el proceso del parto y la participación del acompañante.

Palabras clave: Atención prenatal; Atención Primaria de salud; Enfermería; Parto; Educación para la salud.

\section{Introdução}

O cuidado pré-natal representa a primeira linha de cuidado à saúde da mulher no ciclo gestatório, com atividades direcionadas para as consultas, grupos e visita domiciliares. O pré-natal é o passo elementar para uma gestação, parto e nascimento seguro e saudável, compreendendo não somente os aspectos biológicos/fisiológico, mas também todo contexto biopsicossocial, conforme preconizado pelo Ministério da Saúde (Brasil, 2012; Santos, et al., 2020).

Quando esse cuidado no período gestatório é baseado em evidência científica, pode influenciar para diminuição de morte mortalidade materna, e resultando em benefícios a saúde da mulher e do concepto (Marques, et al, 2021; Martins, et al, 2014).

Desse modo, compreende-se que a assistência prestada no pré-natal é de suma relevância, pois torna-se um momento de criação de vínculo, além de possibilitar o "estímulo e informação sobre os benefícios do parto físiológico, incluindo a elaboração do Plano de Parto", conforme é preconizado nos 10 passos, que se encontram descritos, para uma atenção de qualidade no pré-natal de baixo risco (Brasil, 2012).

Esta ação orienta e estimula o parto normal e a redução de cesarianas desnecessárias, executando-as nas reais indicações. Para preparação do parto e nascimento, a gestante deve receber informações durante todo o curso do pré-natal, de forma positiva e integrativa.

$\mathrm{Na}$ rede básica, a assistência pré-natalista é compartilhada pelos profissionais médicos e enfermeiros, conforme o protocolo do Ministério da Saúde.

Segundo a Lei do Exercício Profissional da Enfermagem e as Diretrizes Curriculares Nacionais, dos Cursos de 
Graduação em Enfermagem, o enfermeiro possui conhecimento técnico-científico para o exercício legal desta assistência, em todo o ciclo gravídico puerperal (Brasil, 1986; Conselho Nacional de Educação, 2001).

O estudo tem por motivação, identificar o que tem produzido em literatura científica sobre a atuação do profissional enfermeiro frente à preparação da mulher gestante para o parto e nascimento. Foi delimitado como objetivo: analisar, em produção científica, ações de enfermagem que contribuem para a preparação da mulher gestante ao parto normal e nascimento.

\section{Metodologia}

O estudo utilizou o método de revisão integrativa. Esse "método de pesquisa objetiva traçar uma análise sobre o conhecimento já construído em pesquisas anteriores sobre um determinado tema" (Botelho, et al, 2011, p.127), viabilizando o aprofundamento do conhecimento sobre a temática investigada.

A revisão integrativa oportuniza a inclusão de diferentes estudos metodológicos para análise e síntese do conhecimento produzida sobre a temática, de forma sistemática e organizada, apontando para o estado da arte, sua implicação no cotidiano e a lacuna do conhecimento (Mendes, Silveira \& Galvão, 2008). Possibilita uma visão ampliada sobre a temática de enfermagem no preparo ao parto normal.

A presente revisão integrativa foi fundamentada em etapas apoiada em Botelho, Cunha, Macedo (2011) descritas a seguir:

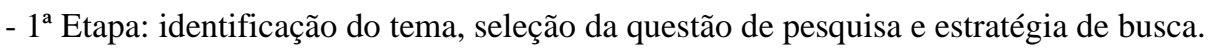

Nesta fase delimitou-se o tema: "Contribuição da enfermagem para a preparação da mulher gestante ao parto normal e nascimento"; e a questão de busca: "Como a literatura aborda as ações do enfermeiro para a preparação da mulher gestante ao parto e nascimento?" Foi elaborado para esta pesquisa os acrônimos da estratégia PICo, onde P: população -Enfermeiro -; I: fenômeno de interesse -ações de enfermagem para a preparação da mulher gestante -; Co: contexto -parto e nascimento -.

Foi visitada a base DESCS - DESCRITORES EM SAÚDE e delimitados: "Cuidado Pré-Natal”; "atenção Primária à Saúde”; "enfermagem”; "parto”; "educação em saúde”. Os descritores apresentam as seguintes definições no DeSC:

$\checkmark$ "Cuidado Pré-Natal": Pré-natal inclui a prevenção, a promoção da saúde e o tratamento dos problemas que possam ocorrer durante o período gestacional e após o parto. A adesão das mulheres ao pré-natal está relacionada com a qualidade da assistência prestada pelo serviço e pelos profissionais de saúde, fator essencial para redução dos elevados índices de mortalidade materna e perinatal. (Assistência Pré-Natal - Ministério da Saúde, 2000)

$\checkmark$ "Atenção Primária à Saúde": É a assistência sanitária essencial baseada em métodos e tecnologias práticas, cientificamente fundados e socialmente aceitáveis, postos ao alcance de todos os indivíduos e famílias da comunidade mediante a sua plena participação e a um custo que a comunidade e o país possam suportar, em todas e cada etapa do seu desenvolvimento, com um espírito de auto-responsabilidade e autodeterminação. (Declaração de Alma-Ata Organização Pan-Americana da Saúde, 2003)

$\checkmark$ "Enfermagem": Campo da enfermagem voltado para a promoção, manutenção e restauração da saúde.

$\checkmark$ "Parto": Processo de nascimento de um ou mais filhos.

$\checkmark$ "Educação em Saúde": A educação em saúde objetiva desenvolver nas pessoas um sentido de responsabilidade, como indivíduo, membro de uma família e de uma comunidade, para com a saúde, tanto individual como coletivamente.

Essa foi realizada em outubro de 2019. A busca de artigos foi realizada nas bases: Literatura Latino-Americana e do Caribe em Ciências da Saúde, Medical Literature Analysis and Retrieval System Online e Bases de dados de enfermagem (LILACS, MEDLINE e BDENF), através da Biblioteca Virtual em Saúde (BVS). A delimitação destas bases de buscas ocorreu, respectivamente, por representarem fontes de informações que abrangem literaturas científicas da América Latina e 
Caribe, e Estados Unidos e mais de 70 países, sendo elas relativas à ciência da saúde e Fonte de informação composta por referências bibliográficas da literatura técnico-cientifica brasileira em Enfermagem. (BVS - ENFERMAGEM).

Foram realizadas as buscas dos artigos na biblioteca virtual em saúde, utilizado os descritores: "Cuidado Pré-natal", "Atenção primária à saúde", "Enfermagem", "Parto Normal” e "Educação em saúde”, com aspas.

Em seguida, realizaram-se as articulações de todos os descritores e em seguida, foram suprimindo um a um.

- $2^{\mathrm{a}}$ Etapa: Estabelecimento de critérios de inclusão e exclusão.

Os critérios de inclusão delimitados para busca em bases de dados foram: artigos originais com rigor metodológico de pesquisa científica; artigos no idioma português Brasil, espanhol e inglês, por serem de fácil acesso aos profissionais de saúde no Brasil. Artigos na íntegra, online e com acesso gratuito; artigos publicados nos anos de 2008 a 2018. Essa delimitação deuse por acreditar-se que apresentam dados atualizados sobre a temática.

O critério de exclusão foi: artigos cujo resumo não aborde a temática trabalhada: ações do enfermeiro para a preparação da mulher gestante ao parto e nascimento. Apesar de terem sido localizados pelos descritores utilizados para este estudo.

Como estratégia de conhecimentos sobre existência de publicações foi realizado a busca das produções através dos descritores individualizados. Desta maneira, foi criado um quadro para demostrar o quantitativo de produções científicas indexados na Biblioteca Virtual em Saúde- BVS.

Quadro 1 - Quantitativo de produções científicas na Biblioteca Virtual em Saúde- BVS.

\begin{tabular}{|l|c|c|c|c|c|c|c|c|}
\hline \multicolumn{2}{|c|}{ Biblioteca Virtual em Saúde - BVS } \\
\hline Descritores & Total & Artigos & $\begin{array}{c}\text { Texto } \\
\text { completo - } \\
\text { disponível }\end{array}$ & $\begin{array}{c}\text { Idiomas } \\
\text { (port., } \\
\text { inglês, } \\
\text { esp.) }\end{array}$ & $\begin{array}{c}\text { Ano } \\
(2008- \\
2020)\end{array}$ & LILACS & MEDLINE & BDENF \\
\hline $\begin{array}{l}\text { "Cuidado } \\
\text { Pré-natal" }\end{array}$ & 736 & 685 & 646 & 645 & 603 & 468 & 4 & 166 \\
\hline $\begin{array}{l}\text { "Atenção } \\
\text { primária } \\
\text { saúde" }\end{array}$ & 88.543 & 79.754 & 34.590 & 34.244 & 32.601 & 5.225 & 26.212 & 1.282 \\
\hline $\begin{array}{l}\text { "Enfermage } \\
\text { m" }\end{array}$ & 582.841 & 566.996 & 126.905 & 124.157 & 115.367 & 17.759 & 87.141 & 14.305 \\
\hline "Parto" & 3.475 & 3.161 & 896 & 892 & 728 & 348 & 230 & 165 \\
\hline $\begin{array}{l}\text { "Educação } \\
\text { em saúde" }\end{array}$ & 170.258 & 142.074 & 43.979 & 43.047 & 39.421 & 6.104 & 30.895 & 1.724 \\
\hline
\end{tabular}

Fonte: Biblioteca Virtual em Saúde (BVS).

Mediante ao levantamento realizado na BVS com os descritores acima apresentados foi possível identificar que existe um quantitativo expressivo de publicações científico nas bases de dados indexados na BVS.

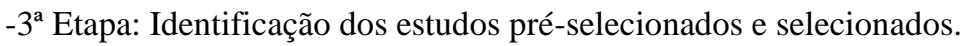

Para organizar as informações foi elaborada uma matriz de análise, apresentando o quantitativo de produções localizadas mediante articulação dos descritores.

No Quadro 2 a seguir, estão esquematizados e descritos os achados frente o quantitativo de produções científicas, que desvelou-se na aplicação dos critérios de inclusão nas três bases de busca. 
Quadro 2 - Quantidade de produções científicas que emergiram mediante o cruzamento de descritores de acordo com critério de inclusão, na base LILACS, MEDLINE E BDENF.

\begin{tabular}{|c|c|c|c|c|c|c|c|c|}
\hline Descritores & Total & Artigos & $\begin{array}{c}\text { Texto } \\
\text { completo- } \\
\text { disponível }\end{array}$ & $\begin{array}{l}\text { Idiomas } \\
\text { (port., } \\
\text { inglês, } \\
\text { esp.) }\end{array}$ & $\begin{array}{l}\text { Ano } \\
(2008- \\
2018)\end{array}$ & LILACS & MEDLINE & BDENF \\
\hline $\begin{array}{l}\text { "Cuidado Pré-natal" and } \\
\text { "Atenção primária à saúde" } \\
\text { and "Enfermagem" and } \\
\text { "parto" and "Educação em } \\
\text { saúde" }\end{array}$ & 2 & 2 & 2 & 2 & 2 & 2 & 0 & 1 \\
\hline $\begin{array}{l}\text { "Cuidado Pré-natal" and } \\
\text { "Atenção primária à saúde" } \\
\text { and "Enfermagem" and } \\
\text { "parto" }\end{array}$ & 3 & 3 & 3 & 3 & 3 & 3 & 0 & 1 \\
\hline $\begin{array}{l}\text { "Cuidado Pré-natal" and } \\
\text { "Atenção primária à saúde" } \\
\text { and "Enfermagem" }\end{array}$ & 38 & 38 & 35 & 35 & 33 & 19 & 0 & 18 \\
\hline $\begin{array}{l}\text { "Cuidado Pré-natal" and } \\
\text { "Atenção primária à saúde" } \\
\text { and "Parto" }\end{array}$ & 19 & 17 & 17 & 17 & 12 & 9 & 0 & 4 \\
\hline $\begin{array}{l}\text { "Cuidado Pré-natal" and } \\
\text { "Atenção primária à saúde" } \\
\text { and "Educação em saúde" }\end{array}$ & 15 & 14 & 13 & 13 & 12 & 7 & 0 & 7 \\
\hline $\begin{array}{l}\text { "Cuidado Pré-natal" and } \\
\text { "Enfermagem" and "Parto" }\end{array}$ & 53 & 45 & 45 & 45 & 43 & 27 & 0 & 21 \\
\hline $\begin{array}{l}\text { "Cuidado Pré-natal" and } \\
\text { "Atenção primária à saúde" } \\
\text { and "Educação em saúde" }\end{array}$ & 20 & 16 & 16 & 16 & 14 & 11 & 0 & 4 \\
\hline $\begin{array}{l}\text { "Cuidado Pré-natal" and } \\
\text { "Parto" }\end{array}$ & 253 & 224 & 212 & 212 & 198 & 167 & 1 & 37 \\
\hline
\end{tabular}

Fonte: Biblioteca Virtual em Saúde (BVS).

Nas bases de dados LILACS e BDENF, evidenciou-se o maior quantitativo de artigos com a articulação dos descritores: "Cuidado Pré-natal" and "Parto". Seguido dos descritores: "Cuidado Pré-natal" and "Enfermagem" and "Parto".

Por meio da associação dos descritores: "Cuidado Pré-natal" and "Atenção primária à saúde" and "Enfermagem" and "parto" and "Educação em saúde", verificou-se um quantitativo baixo de produções.

$\mathrm{Na}$ base de dados MEDLINE, identificou-se uma publicação indexada através do cruzamento dos descritores: "Cuidado Pré-natal" and "Parto". Através da articulação dos outros descritores estabelecidos destaca-se ausência de produções.

Para conhecer o quantitativo de produções científicas foi desenvolvida a busca de cada descritor individualmente. Em seguida, realizaram-se as articulações de todos os descritores. 
Os artigos lidos na íntegra estavam indexados nas bases LILCAS e BDENF. Intitulados como: "Pré-natal: preparo para o parto na atenção primária à saúde no sul do Brasil" (Gonçalves, et al., 2017); "As ações de enfermagem no cuidado à gestante: um desafio à atenção primária de saúde" (Garcia, et al., 2018); "O papel do enfermeiro do programa saúde da família no atendimento pré-natal" (Duarte, S. J. H. \& Almeida, E. P., 2014); "Ações do pré-natal realizadas pela equipe de enfermagem na atenção primária à saúde, Cuiabá” (Duarte, S. J. H \& Mamede, M. V., 2013) ; "Abordagem da equipe de enfermagem acerca do parto humanizado no pré-natal: uma revisão narrativa" (Ragagnin, et al., 2017); "Diálogo com equipes de Saúde da Família sobre parto no pré-natal: uma investigação comunicativa” (Macedo, et al., 2017); "Gestação e preparo para o parto: programas de intervenção" (Silva, 2013).

Quatro artigos foram excluídos, pois não apresentam adequação com a temática do estudo.

Na base de LILACS com os descritores: "Cuidado Pré-natal" and "Atenção primária à saúde" and "Enfermagem" and "Parto" and "Educação em saúde", identificou um artigo, intitulado: "Pré-natal: preparo para o parto na atenção primária à saúde no sul do Brasil" (Gonçalves, et al., 2017). Avalia a relação entre assistência pré-natal e orientações para o parto na Atenção Primária à Saúde. Trata da assistência frente ao preparo ao parto ligado ao cartão de pré-natal e perguntas. Não aborda as ações de enfermagem. O mesmo artigo é localizado nas demais combinações de descritores definidos e descritos no quadro 2.

O segundo artigo: "o papel do enfermeiro do programa saúde da família no atendimento pré-natal” (Duarte, S. J. H \& Almeida, E. P, 2014), tem por objetivo descrever as ações do enfermeiro na atenção pré-natal inserida no Programa Saúde da Família e discutir o cuidado de enfermagem como fundamental ao pré-natal adequado. Não aborda ações de preparo para o parto, abrange a assistência de enfermagem de forma geral, no que tange as orientações prestadas pelo profissional. O presente estudo foi localizado com os descritores "Cuidado Pré-natal" and "Atenção primária à saúde" and "Educação em saúde". Indexado nas bases LILACS e BDENF.

Através dos descritores: "Cuidado Pré-natal" and "Parto" foram apreciados dois artigos. O primeiro: "diálogo com equipes de Saúde da Família sobre parto no pré-natal: uma investigação comunicativa” (Macedo, et al., 2017), visa analisar junto as equipes de saúde da família, os elementos que dificultam a abordagem do parto no pré-natal. Não se adéqua por abordar conhecimento profissional e fragilidade do campo e pessoal para trabalhar o preparo para o parto, não contempla especificamente o fazer do enfermeiro. O segundo artigo: "gestação e preparo para o parto: programas de intervenção" (Silva, E. A. T, 2013). Discorrer sobre os vários modelos de programas de intervenção pré-natal, a partir de resultados encontrados em pesquisas realizadas no doutorado, além de dados da literatura e da experiência clínica da autora. Artigo não selecionado por ser de psicologia e não tratar das ações de enfermagem.

Para exemplificação do fluxo da seleção dos artigos para a composição da revisão integrativa, um fluxograma foi elaborado para melhor compreensão (Figura 1). 
Figura 1. Fluxograma da exemplificação da seleção dos artigos para revisão integrativa.
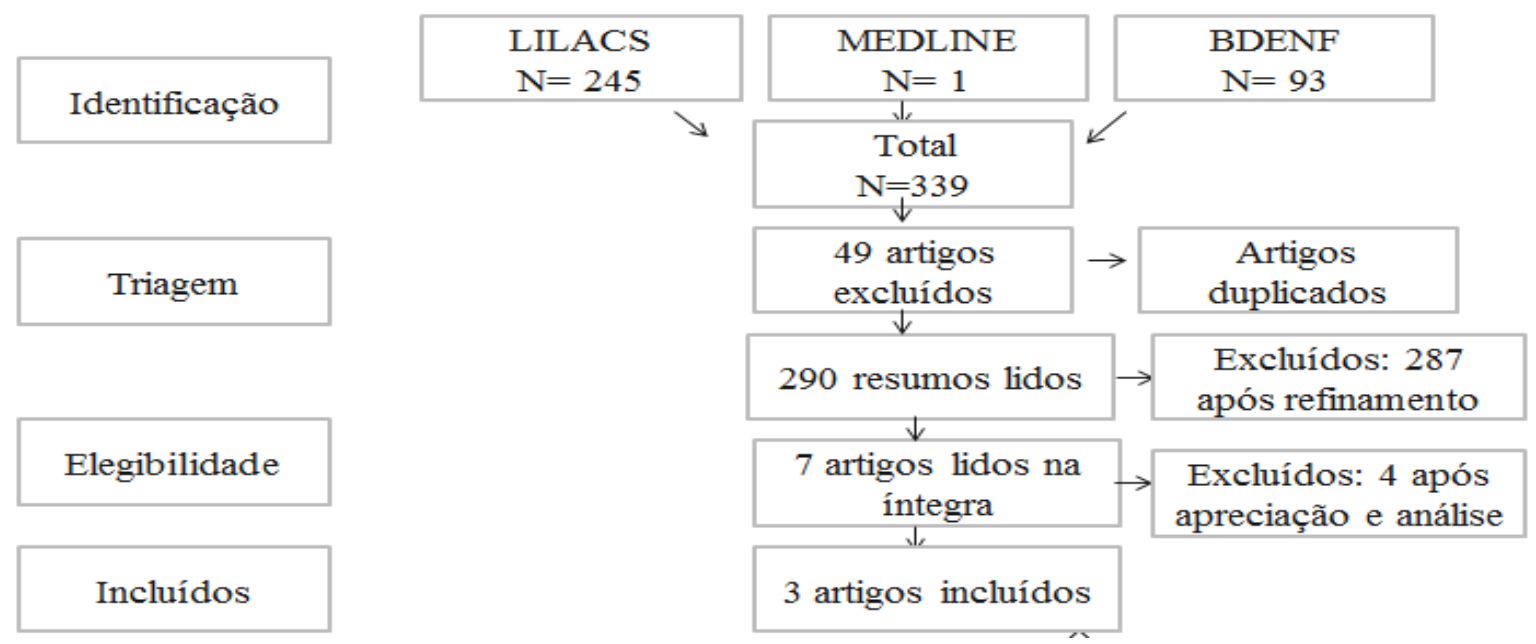

Fonte: Autores.

Da articulação dos descritores frente aos critérios de inclusão identificou-se o quantitativo de 339 artigos; 49 produções foram excluídas por apresentarem amostras duplicadas. Ao realizar a leitura de títulos, resumos, palavras-chave e descritores de todos os 290 artigos localizados, após o refinamento 07 artigos foram lidos na íntegra por serem potencialmente apropriados, porém após apreciação e análise do conteúdo exposto, 03 artigos apresentaram aderência à temática proposta, sendo selecionados para análise e discussão.

- $4^{\text {a }}$ Etapa: Caracterização dos estudos selecionados.

Nesta fase foram colhidas e organizadas as informações dos artigos localizados.

Após realizar a leitura e análise dos artigos quanto à temática contribuição da enfermagem para a preparação da mulher gestante ao parto normal e nascimento, obteve-se o quantitativo de 03 artigos.

Com os descritores: "Cuidado Pré-natal" and "Atenção primária à saúde" and "Enfermagem" localizou-se dois artigos, o primeiro, "As ações de enfermagem no cuidado à gestante: um desafio à atenção primária de saúde (Garcia, et al., 2018)". Objetivou-se verificar as ações desenvolvidas pelos profissionais de enfermagem na assistência às gestantes em unidades de atenção primária à saúde. A mesma publicação está indexada no LILACS E BDENF. O segundo, Ações do prénatal realizadas pela equipe de enfermagem na atenção primária à saúde, Cuiabá (Duarte, S. J. H \& Mamede, M. V., 2013). Propôs descrever as ações realizadas pela equipe de enfermagem na atenção pré-natal no município de Cuiabá, MT.

Mediante os descritores: "Cuidado Pré-natal" and "Enfermagem" and "Parto" emergiu um artigo nomeado como: Abordagem da equipe de enfermagem acerca do parto humanizado no pré-natal: uma revisão narrativa (Ragagnin, et al., 2017). Indexado na LILACS E NA BDENF. Com o objetivo de identificar nas publicações da literatura científica as contribuições desenvolvidas pela equipe de enfermagem acerca das abordagens quanto ao parto humanizado durante as consultas no pré-natal. O artigo também é localizado através do cruzamento dos descritores "Cuidado Pré-natal" and "Parto".

Como estratégia de sistematização das informações, elaborou-se um quadro como subsídio de análise das informações, exposto a seguir no Quadro 3. 
Research, Society and Development, v. 10, n. 10, e228101018672, 2021

(CC BY 4.0) | ISSN 2525-3409 | DOI: http://dx.doi.org/10.33448/rsd-v10i10.18672

Quadro 3. Caracterização, categorização do tipo de estudo, objetivo do estudo e das ações do enfermeiro, dos artigos que atendem a temática

\begin{tabular}{|c|c|c|c|c|c|c|c|c|}
\hline Título do Artigo e Autores & Revista & $\begin{array}{l}\text { Qualis } \\
\text { capes }\end{array}$ & $\begin{array}{c}\text { Ano de } \\
\text { Publicação }\end{array}$ & $\begin{array}{l}\text { Categoria } \\
\text { profissional } \\
\text { do autor }\end{array}$ & Vínculo profissional do autor & $\begin{array}{l}\text { Tipo de } \\
\text { estudo }\end{array}$ & Objetivo do estudo & Ações \\
\hline $\begin{array}{l}\text { As ações de enfermagem } \\
\text { no cuidado à gestante: um } \\
\text { desafio à atenção primária } \\
\text { de saúde } \\
\text { (Garcia, et al., 2018) }\end{array}$ & $\begin{array}{l}\text { Cuidado é } \\
\text { fundamental } \\
\text { online }\end{array}$ & B2 & 2018 & Enfermeira & $\begin{array}{l}\text { Estudante de doutorado na Universidade de São } \\
\text { Paulo. } \\
\text { Mestres pela Universidade Federal de Alfena. } \\
\text { Professor associado do Departamento de enf. } \\
\text { Materno infantil e Saúde Pública de Ribeirão } \\
\text { Preto da Universidade de São Paulo. } \\
\text { Professor adjunto da Escola de Enfermagem da } \\
\text { Universidade de Alfena. }\end{array}$ & $\begin{array}{l}\text { Estudo } \\
\text { descritivo e } \\
\text { transversal }\end{array}$ & $\begin{array}{l}\text { Verificar as ações } \\
\text { desenvolvidas } \\
\text { profissionais de enfermagem } \\
\text { na assistência às gestantes em } \\
\text { unidades de atenção primária } \\
\text { à saúde. }\end{array}$ & $\begin{array}{l}\text { Orientação de trabalho de } \\
\text { parto. }\end{array}$ \\
\hline $\begin{array}{l}\text { Ações do pré-natal } \\
\text { realizadas pela equipe de } \\
\text { enfermagem na atenção } \\
\text { primária à saúde, Cuiabá } \\
\begin{array}{l}\text { (Duarte, S. J. H } \quad \& \\
\text { Mamede, M. V., 2013) }\end{array}\end{array}$ & $\begin{array}{l}\text { Ciencia y } \\
\text { Enfermeria }\end{array}$ & B1 & 2013 & Enfermeira & $\begin{array}{l}\text { Professor Universidade Federal de Mato Grosso } \\
\text { do Sul. } \\
\text { Professor Universidade de São Paulo. }\end{array}$ & $\begin{array}{l}\text { Estudo } \\
\text { descritivo, } \\
\text { exploratório, } \\
\text { transversal, de } \\
\text { abordagem } \\
\text { qualitativa }\end{array}$ & $\begin{array}{l}\text { Descrever as ações realizadas } \\
\text { pela equipe de enfermagem na } \\
\text { atenção pré-natal no } \\
\text { município de Cuiabá, MT. }\end{array}$ & $\begin{array}{l}\text { Orientação quanto sinais } \\
\text { de trabalho de parto. }\end{array}$ \\
\hline $\begin{array}{l}\text { Abordagem da equipe de } \\
\text { enfermagem acerca do } \\
\text { parto humanizado no pré- } \\
\text { natal: uma revisão } \\
\text { narrativa } \\
\text { (Ragagnin, et al., 2017) }\end{array}$ & $\begin{array}{l}\text { Cuidado é } \\
\text { fundamental } \\
\text { online }\end{array}$ & B2 & 2017 & Enfermeira & $\begin{array}{l}\text { Enfermeira. } \\
\text { Pesquisadora do Grupo de Pesquisa em } \\
\text { Empreendedorismo Social de Enfermagem e } \\
\text { Saúde. } \\
\text { Enfermeira. } \\
\text { Residente de Enfermagem Obstétrica. } \\
\text { Enfermeira Obstetra }\end{array}$ & $\begin{array}{l}\text { Revisão } \\
\text { narrativa }\end{array}$ & $\begin{array}{l}\text { Identificar nas publicações da } \\
\text { literatura científica as } \\
\text { contribuições desenvolvidas } \\
\text { pela equipe de enfermagem } \\
\text { acerca das abordagens quanto } \\
\text { ao parto humanizado durante } \\
\text { as consultas no pré-natal. }\end{array}$ & $\begin{array}{l}\text { Informação da tecnologia } \\
\text { do cuidado e da escolha do } \\
\text { parto pela enfermagem. } \\
\text { Oficinas educativas com } \\
\text { dramatizações } \\
\text { relacionadas à gestação e } \\
\text { ao trabalho de parto. }\end{array}$ \\
\hline
\end{tabular}

Fonte: Biblioteca Virtual de Saúde (BVS) 


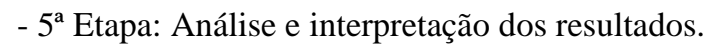

Esta etapa ocorre mediante a discussão dos três artigos selecionados supracitados, e por meio de dados inseridos na matriz de análise, tendo por embasamento a técnica de análise de conteúdo (Bardin, 2011).

- $6^{a}$ Etapa: Análise e síntese do conhecimento

Nesta última fase, foi desenvolvida a síntese do conhecimento sobre as ações dos enfermeiros para a preparação da mulher ao parto e nascimento.

\section{Resultados e Discussão}

Pôde-se evidenciar que os três artigos foram publicados em revistas científicas de enfermagem, nos anos de 2013, 2017 e 2018, com classificação B1 e B2 pela qualis capes, por profissionais de saúde vinculados a instituições de pesquisa.

A metodologia dos estudos utilizada foi: descritivo e transversal, descritivo, exploratório, transversal e revisão narrativa.

Destacam-se como ações do enfermeiro: orientação quanto ao local e serviços de atendimento ao parto, mudanças corporais, aleitamento materno, cuidado com o RN, sinais de trabalho de parto, tecnologia do cuidado e da escolha do parto pela gestante e oficinas educativas com dramatizações relacionadas à gestação e ao trabalho de parto. Essas ações apontaram para uma categoria analítica: atividades educativas em saúde para o preparo do parto.

Mesmo que as ações de enfermagem sejam evidenciadas nas publicações científicas, o olhar do enfermeiro está direcionado: a referência da mulher à maternidade, aleitamento materno, e cuidados e procedimentos específicos com o recémnascido, como estratégia de cuidado às orientações e oficinas educativas. Apesar das ações descritas em artigos, os mesmos apontam como essas práticas têm alcançado as gestantes dos estudos.

Conforme Duarte e Mamede (2013), do quantitativo total de 74 gestantes participantes, no que tange as orientações prestadas totalizam: "local e serviços de atendimento ao parto $56.7 \%$, cuidados com o recém-nascido $17,5 \%$, aleitamento materno 9.4\%, sinais de trabalho de parto 9.4\% e modificação corporais 6.7\%” (p. 120-121). Já os autores Garcia, et al. (2018), no total de 134 participantes apresentam a frequência das orientações fornecidas totalizando: "quando procurar o serviço de saúde $5.22 \%$, aleitamento materno $30.59 \%$, sinais do trabalho de parto $4.47 \%$, teste do pezinho $21.64 \%$ e cuidados com o RN 8.94\%” (p. 866). No artigo dos Ragagnin, et al. (2017) não aponta o quantitativo dos sujeitos participantes dos estudos por ser uma revisão narrativa.

Frente aos dados apresentados das investigações científicas, existem informações sendo fornecidas às gestantes, porém não existe uma expressividade no total de todas as informações podendo concluir que nem todas as gestantes recebem todas as orientações para o preparo para o parto e nascimento.

(...) Planejamento individual, considerando-se o local, o transporte, os recursos necessários para o parto e para o recém-nascido, o apoio familiar e social; Orientações e incentivo para o parto normal, resgatando-se a gestação, o parto, o puerpério e o aleitamento materno como processos fisiológicos; Incentivo ao protagonismo da mulher, potencializando sua capacidade inata de dar à luz; Orientação e incentivo para o aleitamento materno e orientação específica para as mulheres que não poderão amamentar; Importância do planejamento familiar num contexto de escolha informada, com incentivo à dupla proteção; Sinais e sintomas do parto; Cuidados após o parto com a mulher e o recém-nascido, estimulando o retorno ao serviço de saúde; Saúde mental e violência doméstica e sexual; Benefícios legais a que a mulher tem direito, incluindo a Lei do Acompanhante; Impacto e agravos das condições de trabalho sobre a gestação, o parto e o puerpério. O estabelecimento do vínculo entre pai e filho, fundamental para o desenvolvimento saudável da criança; $\mathrm{O}$ direito a acompanhante de sua escolha durante o trabalho de parto, no parto e no pós parto, garantido pela Lei $\mathrm{n}^{\circ} 11.108$, de 7 de abril de 2005, regulamentada pela Portaria MS/GM no 2.418 , de 2 de dezembro de 2005; Gravidez na adolescência e dificuldades sociais e familiares; Importância das consultas puerperais; Cuidados com o recém-nascido; Importância da realização da triagem neonatal (teste do pezinho) na primeira semana de vida do recém-nascido; Importância do acompanhamento do crescimento e desenvolvimento da 
criança e importância das medidas preventivas (vacinação, higiene e saneamento do meio ambiente) (Brasil, 2012, p. 147-148).

Afirma também que devem ser prestadas as orientações para as gestantes quanto aos temas: "processo gestacional, mudanças corporais e emocionais, trabalho de parto, parto e puerpério, cuidados com o recém-nascido e amamentação, anatomia e fisiologia maternas, os tipos de parto, participação ativa no nascimento, sexualidade e outras" (Brasil, 2001, p. 2627).

Evidencia-se a importância da troca de saberes e conhecimentos durante todas as consultas de pré-natal, possibilitando a mulher gestante tempo oportuno para compreensão das temáticas sobre o ciclo gravídico puerperal ajudando significativamente a essa mulher a vivenciar uma experiência positiva em seu parto e nascimento de seu bebê.

Desta forma, ao pensar em troca de saberes entre profissionais-mulheres-familiares, as rodas de gestantes destacam-se com um ambiente propício para as mulheres falarem sobre sua experiência/ vivência, medos e anseios e ter seu lugar de fala entre seus pares (Souza, et al., 2020; Ferreira, et al., 2021). Deste modo, o grupo impactará positivamente para o empoderamento da mulher gestante como cidadã de direitos e escolhas.

O Ministério da Saúde (2001) preconiza que o preparo para o parto deve ter abordagem que vise proporcionar a mulher gestante experiência positiva no processo de parturição e se sinta empoderada e dona de todas as etapas do trabalho de parto e parto (Brito, et al, 2015). Neste sentido, preparar para o parto normal é pensar nesta mulher com um olhar nas necessidades de saúde, contemplando os aspectos biopsicossociais e não somente o biológico, ao olhar essa mulher como o todo, pensando em uma assistência ampliada que tem como foco mais que o parto e sim as necessidades como mulher, gestante e puérpera.

No que tange a mulher gestante e seus familiares, Duarte \& Mamede (2013) apontam que a assistência não deve estar somente direcionada aos procedimentos clínicos, mas às ações direcionadas "a promoção de sua saúde, por meio da educação em saúde, do acolhimento, do vínculo de confiança, entre outras tecnologias de forma a desenvolver a autonomia da mulher para o autocuidado" (p. 118).

Vale destacar, que a rede de apoio dessa gestante deve ser inclusa nas ações de pré-natal, trazendo esse acompanhante para o sistema de saúde, ele também poderá receber informações e conhecimentos que repercutirão futuramente no processo de trabalho de parto e parto. O acompanhante é de suma importância nos desfechos positivos de parto e nascimento, pois ao ter um acompanhante informado e participativo, ele ajudará a mulher e ter apoio emocional, segurança e diminuição de medos e anseios (Gomes, et al., 2019).

Os enfermeiros devem direcionar sua assistência à mulher gestante visando o fortalecimento das informações prestadas a essas gestantes, no momento de muitas mudanças não só no aspecto físico como também no emocional, e de estruturação profissional e pessoal, pois o preparo para o parto gera "resultado satisfatório na hora do parto e no pós-parto e auxiliam nos cuidados e no desenvolvimento da criança, mulheres que participam do preparo conseguem gerenciar melhor a ansiedade, medo, estresse do parto e dos cuidados do bebê" (Silva, 2013, p. 208).

Sendo assim, o profissional enfermeiro tem que estar preparado para proporcionar a essa cliente informações baseadas em evidências científicas, facilitando a autonomia, escolha e transmitindo segurança no período gestatório. Segundo o Ministério da Saúde (2001) "o parto precisa ser resgatado como o momento do nascimento, respeitando todos os seus significados devolvendo à mulher o seu direito de ser mãe com humanidade e segurança" (p. 25).

\section{Conclusão}

O estudo desenvolvido evidenciou que os artigos localizam as ações do enfermeiro para a preparação do parto, porém 
observou-se que são pontuais frente ao alcance total da gestante ao conhecimento fornecido, direcionando as informações ao local da maternidade pertencentes, aleitamento materno e cuidados com RN.

Entende-se que existe orientação para preparação para o parto, porém nem todas as clientes recebem todo o conhecimento conforme preconizado pelo Ministério da Saúde.

Mesmo compreendendo que a preparação para o parto possibilitará toda diferença na vida da gestante e em sua família, já que irá proporcionar a diminuição das alterações mentais (como o medo, ansiedade, entre outros), fortalecerá a escolha pela via de parto e outros, visando um atendimento diferenciado, humano e permeado de direitos sociais.

Faz-se necessária a aproximação e o reconhecimento das ações desenvolvidas na assistência do enfermeiro para a preparação ao parto, visando localizar as ações que faltam ser trabalhadas, para criar um plano de ação para se apropriar do conhecimento e do compartilhar, objetivando o fortalecimento frente ao conhecimento para gestante.

Diante do exposto, torna-se relevante o desenvolvimento da temática e o aprofundamento de investigações que contemplem o assistir do enfermeiro na preparação para o parto.

Neste entendimento, sugerimos para futuros estudos diversidade de produções científicas que abordem a temática de preparação para o parto no ciclo gestatório frente à atuação do profissional enfermeiro.

\section{Referências}

Bardin, L. (2010). Análise de conteúdo. (70a ed.), Edições.

Brasil. (2012). Ministério da Saúde. Atenção ao pré-natal de baixo risco. Ministério da Saúde.

Brasil. (1986). Lei no 7.497 de 25 de junho de 1986 (Br). Dispõe sobre a regulamentação do exercício da enfermagem, e dá outras providências. Presidência da República, http://www.cofen.gov.br/lei-n-749886-de-25-de-junho-de-1986_4161.html

Brasil. (2001). Ministério da Saúde. Parto, aborto e puerpério: assistência humanizada à mulher. Ministério da Saúde.

Brasil. (2019). Portal regional da bvs: informação e conhecimento para Saúde. pesquisa.bvsalud.org

Brito, C. A. B, Silva, A. S. S, Cruz, R. S. B. L. C., \& Pinto, S. L. (2015) Percepção de puérperas sobre a preparação para o parto no pré-natal. Revista da Rede de Enfermagem do Nordeste, 16(4), 470-478. 10.15253/2175-6783.2015000400003

Botelho, L. L. R., Cunha, C. C. de A., \& Macedo, M. (2011). Método da revisão integrativa nos estudos organizacionais. Gestão e Sociedade, 5(11), 121-136. doi: https://doi.org/10.21171/ges.v5i11.1220

Conselho Nacional de Educação (2001). Resolução nº. 3 de 7 de novembro de 2001 (Br). Institui Diretrizes Curriculares Nacionais do Curso de Graduação em Enfermagem. Diário Oficial da União, http://portal.mec.gov.br/cne/arquivos/pdf/CES03.pdf

DESC (2019). Descritores em ciência da saúde. https://decs.bvsalud.org/

Duarte, S. J. H. \& Mamede, M. V. (2013). Ações do pré-natal realizadas pela equipe de enfermagem na atenção primária à saúde, Cuiabá. Ciencia y Enfermería, 11(1), 117-129. https://www.redalyc.org/pdf/3704/370441812011.pdf

Duarte, S. J. H. \& Almeida, E. P. (2014). O papel do enfermeiro do programa saúde da família no atendimento pré-natal. Revista de Enfermagem do Centro Oeste Mineiro, 4(1), 1029-1035. https://doi.org/10.19175/recom.v0i0.137

Ferreira, G. E., Fernandes, I. T. G. P., Flores, P. C. B., Conceição, K. M, \& Caetano, S. A. (2021). A atenção do enfermeiro na assistência ao pré-natal de baixo risco. Brazilian Journal of Health Review, 4(1), 2114-2127. 10.34119/bjhrv4n1-172

Garcia, E. S. G. F., Bonelli, M. C. P., Oliveira, A. N., Clápis, M. J., \& Leite, E. R. P. C. (2018). As Ações de Enfermagem no Cuidado à Gestante: Um Desafio à Atenção Primária de Saúde. Revista Online de Pesquisa Cuidado é Fundamental (online), 10(3), 863-870. 10.9789/2175-5361.2018.v10i3.863-870

Gomes, I. E. M, Padoin, S. M. M, Langendorf, T. F., Paula, C.C, Gomes, C. A., \& Ribeiro, A. C.(2019). Benefício da presença do acompanhante no processo de parto e nascimento: revisão integrativa. Revista de Enfermagem da UFSM, 61(9), 1-18. 10.5902/2179769234170

Gonçalves, M. F., Teixeira, E. M. B., Silva, M. A. S., Corsi, N. M., Ferrari, R. A. P, Pelloso, S. M., \& Cardelli, A. A. (2017). Pré-natal: preparo para o parto na atenção primária à saúde no sul do Brasil. Revista Gaúcha de Enfermagem (online), 38(3), e2016-0063. https://doi.org/10.1590/1983-1447.2017.03.2016-0063

Marques, B. L., Tomasi, Y. T., Saraiva, S. S., Boing, A. F., \& Geremia, D. S. (2021). Orientações às gestantes no pré-natal: a importância do cuidado compartilhado na atenção primária em saúde. Escola Anna Nery Revista de Enfermagem, 25(1), 1-8. https://doi.org/10.1590/2177-9465-EAN-2020-0098

Martins, A. L., Leite, A. J. M., Friche, A. A. L., Pereira, A. P. E., Gonçalves, A. C., Tuesta, A. A., et al. (2014). Nascer no Brasil: sumário executivo temático da pesquisa. ENSP/Fiocruz. ENSP/Fiocruz. http://www.ensp.fiocruz.br/portal-ensp/informe/site/arquivos/anexos/nascerweb.pdf 
Research, Society and Development, v. 10, n. 10, e228101018672, 2021

(CC BY 4.0) | ISSN 2525-3409 | DOI: http://dx.doi.org/10.33448/rsd-v10i10.18672

Macedo, L. P., Fabbro, M. R. C., Bussadori, J. C. C., \& Izidoro, G. (2017). Diálogo com equipes de Saúde da Família sobre parto no pré-natal: uma investigação comunicativa. Aquichán, 17(4), 413-424. https://doi.org/10.5294/aqui.2017.17.4.5

Mendes, K. D. S., Silveira, R. C. C. P., \& Galvão, C. M. (2008). Revisão integrativa: método de pesquisa para a incorporação de evidências na saúde e na enfermagem. Texto Contexto Enferm [internet], 17 (4), 758-764. https://www.scielo.br/j/tce/a/XzFkq6tjWs4wHNqNjKJLkXQ/?lang=pt\&format=pdf

Ragagnin, M. V., Marchiori, M. R. C. T., Diaz, C. M. G., Nicolli, S. B. P., \& Silva, L. D. (2017). Abordagem da equipe de enfermagem acerca do parto humanizado no pré-natal: uma revisão narrativa. Revista Online de Pesquisa Cuidado é Fundamental (online), 9(4), 1177-1182. 10.9789/21755361.2017.v9i4.1177-1182

Santos, L. L., Oliveira, R. C. M., Barbosa, V. V. S., \& Ribeiro, A. S. (2020). Papel do Enfermeiro na assistência do pré-natal na Atenção Básica de Saúde. Research, Society and Development, 9(8), e753096202. https://doi.org/10.33448/rsd-v9i8.6202

Silva, E. A. T. (2013). Gestação e preparo para o parto: programas de intervenção. O mundo da Saúde, 37(2), 208-215. http://www.saocamilosp.br/pdf/mundo_saude/102/10.pdf

Sousa, A. J. C., Silva, M. A. M, Nour, G. F. A., Moreira, A. C. A., Freitas, C. A. S. L., Sousa, C. M. F., \& Silva, A. C. O. (2020). Desafios dos enfermeiros na preparação de casais para o parto. Research, Society and Development, 9(12), e1299129137. https://doi.org/10.33448/rsd-v9i12.9137 